\title{
Efficacy of Three-Dimensional Contrast-Enhanced Magnetic Resonance Angiography (3D CE-MRA) in the Diagnosis of Thoracic Outlet Syndrome
}

\author{
Ercüment Ünlü', Derya Demirbağ Kabayel ${ }^{2}$, Ferda Özdemir ${ }^{2}$, Bekir Çağlı' ${ }^{1}$, Sedat A. Tuncel ${ }^{1}$ \\ ${ }^{1}$ Department of Radiology, Faculty of Medicine, Trakya University, Edirne, Turkey \\ ${ }^{2}$ Department of Physical Medicine and Rehabilitation, Faculty of Medicine, Trakya University, Edirne, Turkey
}

\begin{abstract}
Objective: The purpose of this study is to evaluate the effect of various upper extremity positions (adduction-abduction) on vascular structures in contrast-enhanced three-dimensional MR angiographic studies performed in patients with thoracic outlet syndrome.

Materials and Methods: Twenty-two consecutive patients with clinical symptoms of neurovascular thoracic outlet syndrome were examined by $1.0 \mathrm{~T}$ MR unit. Examinations were studied by three-dimensional contrast-enhanced MR angiography with the arms positioned in abduction and adduction in the same patients.

Results: In twenty-one of 44 subclavian arteries, impingement or stenosis with different degrees were found. Majority of lesions were localized in the costoclavicular region. Venous phase sequences of contrast-enhanced MR angiography showed compression of the subclavian vein in the 17 areas.

Conclusion: Thoracic outlet syndrome remains controversial in both diagnosis and treatment, particulary in patients with no muscle atrophy, hand ischemia findings or venous stasis symptoms. Three-dimensional contrast-enhanced MR angiography is noninvasive and requires neither ionizing radiation nor administration of iodinated contrast material- and may be used to diagnose early compression findings and stenosis of the subclavian vessels.
\end{abstract}

Key Words: Thoracic outlet syndrome, contrast-enhanced, three dimensional, magnetic resonance angiography, adduction, abduction

Received: 26.01 .2010

Accepted: 07.06.2010

\section{Introduction}

The term thoracic outlet syndrome (TOS) was used to describe compression of the brachial plexus or subclavian vessels in their courses from the cervical area toward the axilla and proximal arm either at the interscalene triangle, the costoclavicular triangle, or the subcoracoid space (Figure 1). Its clinical picture ranges from severe compression with permanent vascular and/or nervous lesions to intermittent postural symptoms without any organic damage (1-3). TOS was divided into three groups by Wilbourn (4) such as true neurogenic, vascular (either artery and/or vein), and disputed or nonspecific TOS. The last term was defined as a chronic pain syndrome with subtle features suggestive of brachial plexus involvement and, as its etiology was obscure, it was difficult to diagnose. According to literature data, in $98 \%$ of all patients with TOS, the symptoms were attributed to entrapment of the brachial plexus at the thoracic outlet and the cause was vascular compression in only $2 \%(5,6)$. However, because the vessels and nerves were in close proximity to one another in the thoracic outlet, symptoms of vessel abnormalities and neurological signs may coexist in the same patient (7). TOS is more commonly observed in women, and the onset of symptoms usually occurs between the ages of 20 and $50(4,7)$. Thoracic outlet may constrict due to bone structure anomalies such as cervical rib, abnormal first rib, a prominent transverse process of the $7^{\text {th }}$ vertebra or soft tissue abnormalities such as congenital bands and ligaments and scalene muscle hypertrophies. The usual symptoms are shoulder stiffness-weakness, arm-back pain, arm numbness, tingling, coldness and swelling of the extremity $(1,2,8)$.

As there is no generally accepted protocol for the investigation of TOS, diagnosis and treatment involves many physicians and surgeons (5, 7-10). Diagnostic criteria of TOS were reported by Tateishi (11) as symptoms of neurovascular compression in the upper arm regularly or intermittently, positive results in the dynamic provocative tests, and exclusion of the other diseases, including cervical vertebral disorder and peripheral nerve diseases (12). However, clinical diagnosis is often difficult, requiring the use of imaging procedures. Diagnosis is usually made by a combination of physical examination (history and provocative tests) and one or more diagnostic modalities (radiography, electrodiagnostic tests, brachial plexus neurography, color doppler sonography, computed tomography (CT), magnetic resonance (MR) and imaging and digital subtraction angiography (DSA)) $(3,5,7-10,12-27)$. On the other hand, there has recently been considerable disagreement among examiners regarding diagnostic methods of TOS. This is mainly related to the lack of specific objective 
diagnostic tests for early accurate recognition of the neurovascular structures that are compressed $(7,8,17)$.

The usefulness of MR imaging for the assessment of the thoracic outlet and brachial plexus has yet to be fully defined $(3,10,19,20)$. However, it is also important to confirmed or rule out coexisting vascular TOS. Furthermore, little has been written about the diagnostic capability of the contrast-enhanced MR angiography (MRA) by using provocative maneuvers (22-24).

The purpose of this study is to evaluate the effect of the different upper extremity positions (adduction-abduction) on vascular structures in contrast-enhanced three-dimensional (3D) MR angiographic studies performed in patients with TOS.

\section{Material and Methods}

We evaluated twenty-two consecutive patients with clinical symptoms suggestive of TOS by contrast enhanced MRA from August, 2004 to March, 2005. Demographic properties (sex, weight, height, body-mass indexes (BMI)) of the participant patients were determined.

Clinical diagnosis was supported by provocative clinical tests (Adson's, Wright's, Roos's tests) and the patients who produced symptoms in at least two provocative tests were included in the study. Cervical spine and chest radiographs were obtained routinely to rule out other pathologies such as degenerative musculoskeletal diseases and possible upper lung diseases. None of the patients had undergone previous surgery of the upper thoracic region before MR studies. Patients who had thoracal kyphosis at physical examination were excluded from the study. Patients with BMI over 25 (obese patients) also were not included We obtained informed consent from all the patients.

Contrast-enhanced MRA was obtained using the 1-T system (Magnetom Expert; Siemens, Erlangen, Germany) with $20 \mathrm{mT} / \mathrm{m}$ maximum gradient strength. All examinations were performed with a standard body coil. Patients were positioned with a 20-gauche intravenous catheter inserted into the antecubital vein. The first acquisition was done with both arms in adduction (the neutral position) during breath hold. After scout images, the following sequence was programmed. Contrast-enhanced MRA was performed in the coronal plane during injection of $0.2 \mathrm{mmol} / \mathrm{kg}$ Gadolinium diethylenetriaminepentaacetate (Magnevist; Schering, Berlin, Germany), at a rate of $2 \mathrm{~mL} / \mathrm{sec}$ approximately and "chased" with a 20-mL saline injection manually. We used a 3D fast imaging with steady precession (FISP) sequence (5.4/2.2 [TR/TE]; flip angle, $25^{\circ}$; matrix, 110x256; acquisition, 1; field of view, $500 \mathrm{~mm}$; slices, 24-26 of 2-2.5-mm thickness; imaging time, $17 \mathrm{~s}$ ) and the same process was repeated three times. Three acquisitions were performed, first for the arterial phase, second for the equilibrium phase, and lastly for the venous phase. Segmented maximum intensity projection (MIP) images were also obtained for each study with rotation through $180^{\circ}$ at $15^{\circ}$ increments.

The same imaging protocol was obtained with the patient's arms elevated above the head (approximately $130^{\circ}$ of hyperabduction with external rotation) (the postural maneuver). The average total imaging time was $20 \mathrm{~min}$.
No significant complications occurred during or after MRA in any of the patients in this study. Conventional angiography was not performed in any patient.

\section{Results}

Forty-four arms were evaluated in 22 patients. 17 (77.3\%) were women, and 5 (22.7\%) were men. Mean age was 37.4 for women, 43.8 for men. The mean BMI was calculated as 24.2 \pm 0.8 ( $\min : 22.0, \max : 25$ ) and $22.8 \pm 0.8$ ( $\min : 22.8$, max: 24.8) for women and men patients respectively. As a whole, the mean BMI was $24.1 \pm 0.8$ (min: 22.0, max: 25).

There were bilateral symptoms in 13 and unilateral in 9 cases. The symptoms existed in 35 of 44 arms and 18 of them were on the right side and 17 on the left. Subscapular, scapular, and cervical pain in one or both arms, and numbness and weakness of the upper extremity were predominant symptoms in our patients. Complaints were usually seen in the patients when sleeping, the arms above the head and elbows in the flexion position. None of the patients had motor or sensory deficiency or lack of reflexion. Muscular atrophy, ischemia or vascular obstruction of hands and upper extremity, were not seen in any of the patients in the physical examination. 13 osseous anomalies were depicted in 9 of 22 cases. Apophysomegaly of the seventh cervical vertebra was seen in 11 and cervical rib in 2 sides.

No motion-related artifact was seen in MRA. Venous overlapping was seen in the arterial phase in only 3 cases, but it did not create any diagnostic difficulty. There was focal dilatation in 3, and equivocal impingement findings in 6 of 44 sides in MRA images obtained in the adduction position. No significant venous compression findings were found in adduction. There was impingement or stenosis of less than $60 \%$ in 19 of 44 sides in MRA images obtained in the abduction position. Stenosis of more than $60 \%$ was obtained in only two cases. Pathologic appearance was seen in the costoclaviculer region, interscalene triangle, retropectoralis minor space, and in 17, 2, 2 cases, respectively. Of the arterial pathologies t obtained at abduction, 14 were on the right, and 9 were on the left. Subclavian venous compression findings during abduction were found in 17 of 44 sides, however, thrombosis was not determined in any of the cases. As venous pathologies coexist with arterial lesions in 14 sides, isolated venous compression findings were found in 3 sides, in MRA images obtained in the abduction position.

Representative cases are illustrated in figures 2-9.

\section{Discussion}

One of the most controversial clinical subjects in medicine is TOS. The clinical existence of TOS results from compression or irritation of the neurovascular bundle at the cervicothoracicbrachial plexus. The clinical syndrome may be isolated to one or a mixture of these compressed anatomic structures $(1,2,7)$. For patients in whom TOS is suspected, extensive provocative maneuvers and additional technical examinations must be performed to establish a differential diagnosis or to confirm the need for treatment $(2,7,23)$. As symptoms are indefinite and 


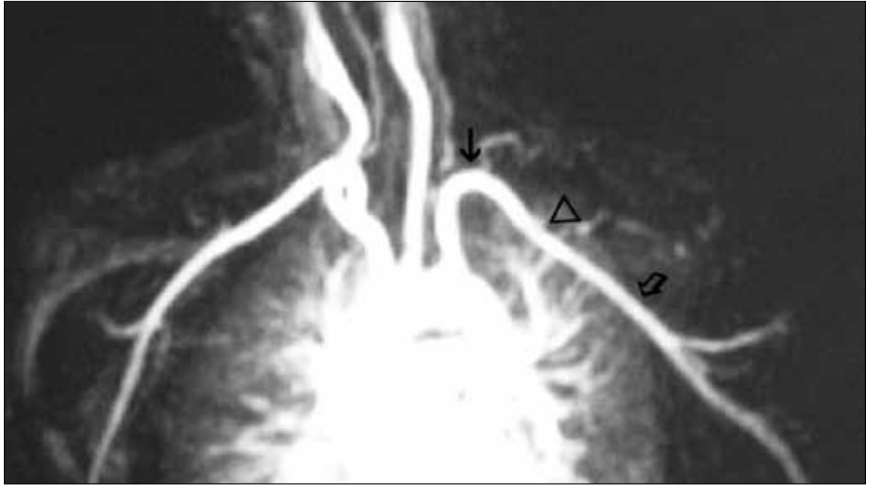

Figure 1. The projections of interscalene triangle (black arrow), costoclavicular region (arrowhead) and subcoracoid space (open arrow) was seen on subclavian artery tract

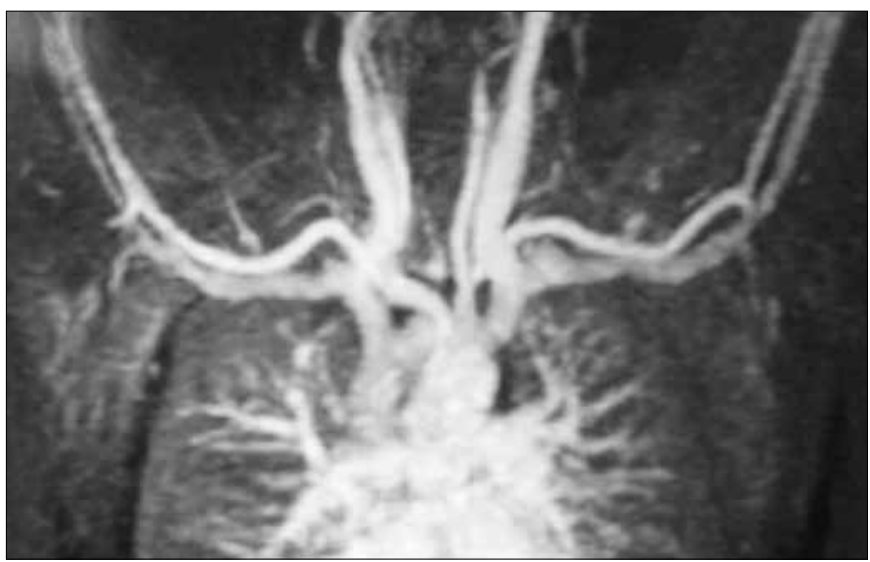

Figure 2. 3D contrast-enhanced MRA in equilibrium phase makes it possible to examine the arterial and venous findings obtained during arms abduction

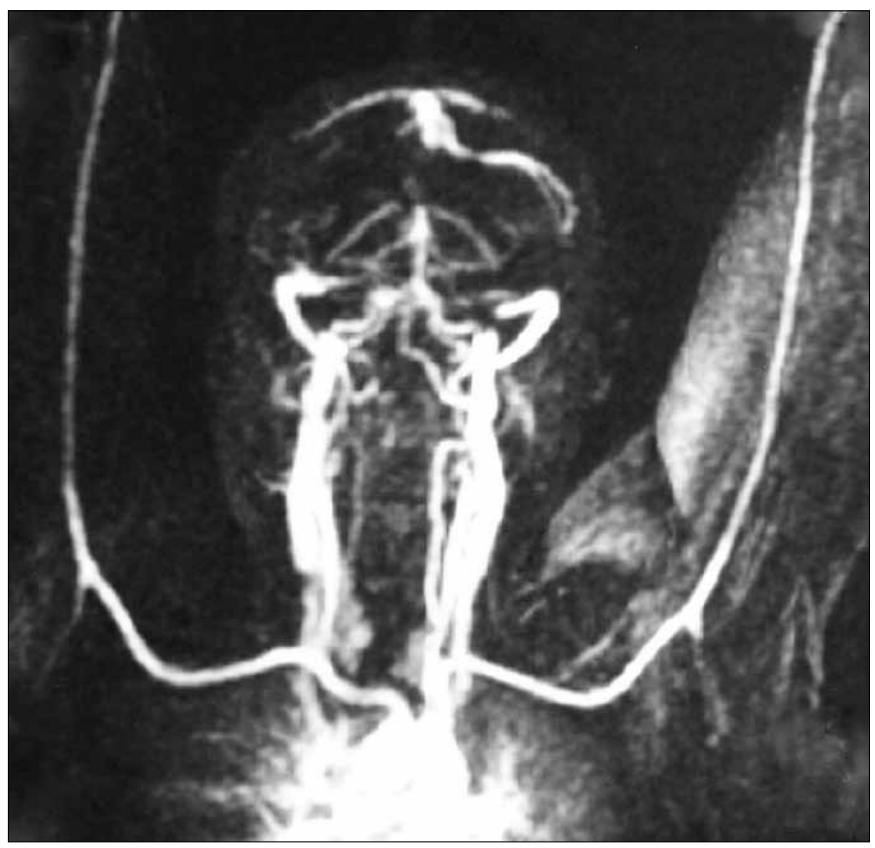

Figure 3. It was possible to show the area between arcus aorta and antecubital fossa in abduction by 3D contrastenhanced MRA

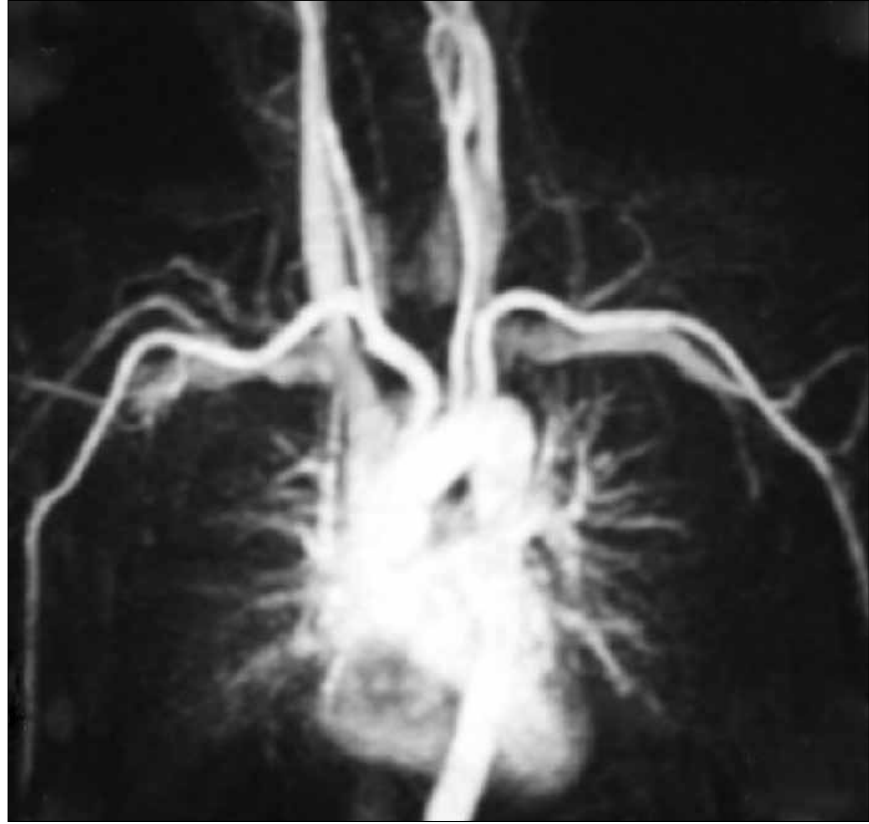

Figure 4. Undulation sign due to possible structural postural abnormality was realized in right subclavian artery during adduction by MRA

nonspecific in many cases, imaging is required to explain the reason and location in order to provide information for treatment. However, several clinical, radiological and electrodiagnostic tests have been described in the diagnosis of TOS, and there is no quantifiable test accepted as the "gold standard". Although provocative positioning tests are usually applied in the diagnosis of TOS, some studies have shown that positive results can also be obtained in the normal subjects. Also, the false positive and negative ratio is found to be quite high in these tests $(6,8,17)$. Abnormal anatomy at the thoracic outlet often causes TOS. The incidence of anomalous cervical ribs is $0.17 \%$ to $0.74 \%$ in the general population and approximately $6 \%$ to $11 \%$ in patients with TOS $(1,2,8)$. Cervical rib was seen in two $(9.09 \%)$ of 24 patients in our study.

Although arterial or venous vascular complications resulting from thoracic outlet compression are rare, they can be significant because of the threat of ischemia and gangrene of the limb. In management, early diagnosis of lesions resulting from compression or irritation of vascular structures can be important, because advanced lesions including significant arterial stenosis, arterial aneurysm and venous thrombosis may present with complications such as distal emboli, limb ischemia or also cerebral ischemia. Also, in these cases that are too late for conservative therapy, invasive therapeutic approaches (surgical, endovascular treatment) are generally required $(5,26-28)$. For these reasons, early diagnosis of vascular TOS requires appropriate scanning methods.

In clinical practice, after a detailed examination and plain radiographs of the relevant area, traditional catheter angiography has been used to diagnose vascular abnormalities related with TOS. Catheter angiographic findings were described, such as mild dilatation of the subclavian artery, which is the common finding of the abnormal course of the distal subclavian artery, and also stenosis and aneurysmatic dilatation in TOS 

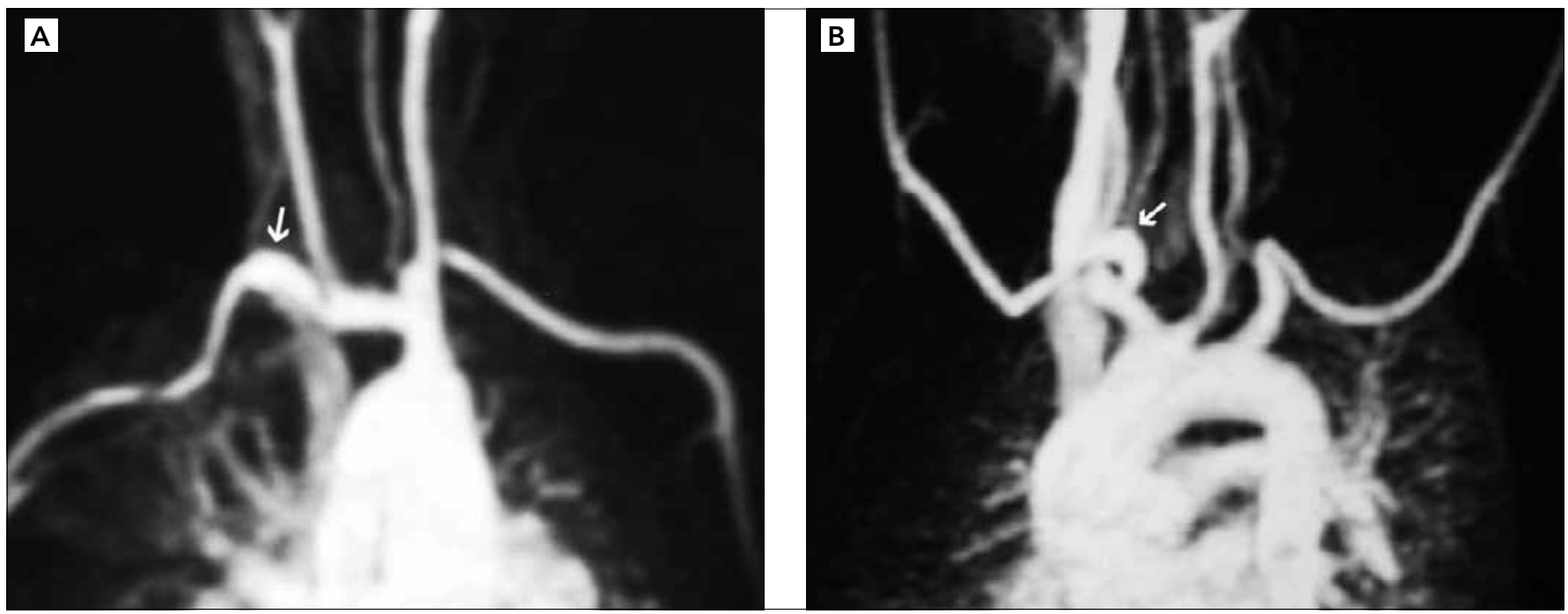

Figure 5. On MRA images during arm adduction (A) and abduction (B) fusiform dilatation (arrows) was determined in proximal part of right subclavian artery
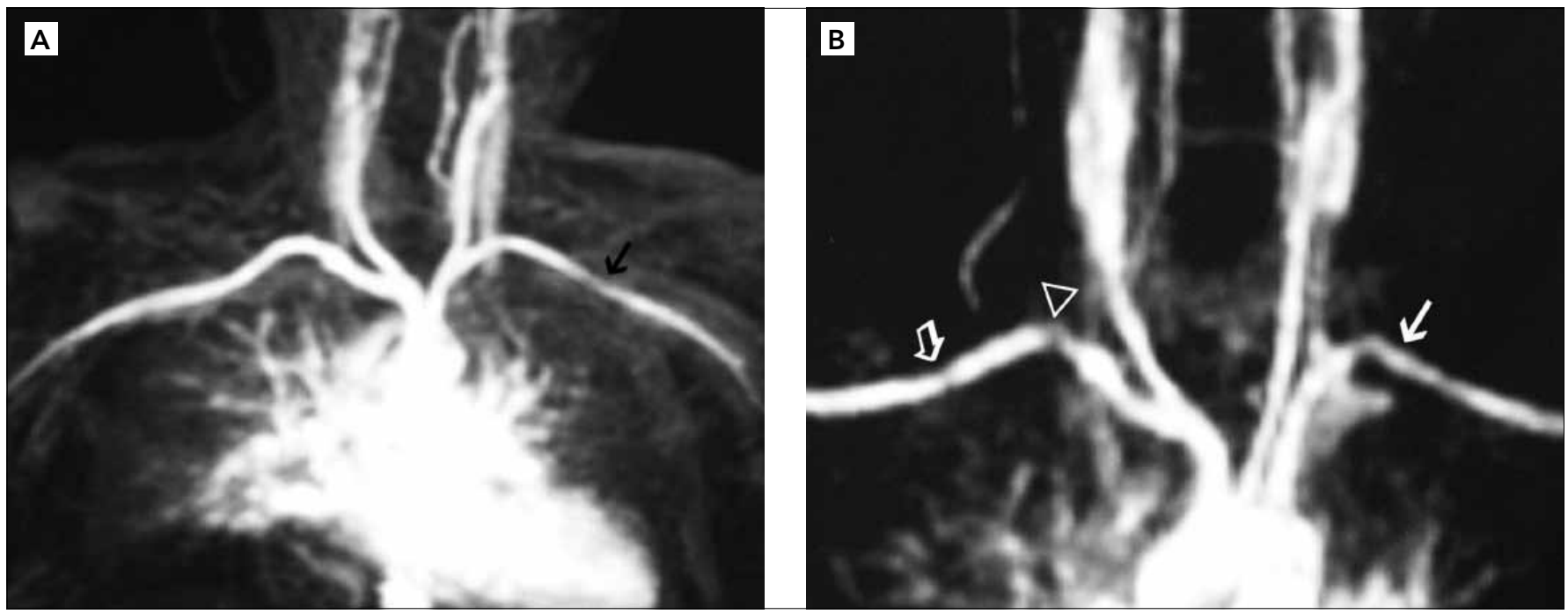

Figure 6. (A) During adduction at left costoclavicular region in subclavian artery minimal lack of calibration (arrow) was obtained by MRA. (B) Although during abduction, stenosis was more evident at left subclavian artery (solid arrow), impingement finding was seen at right interscalene triangle (arrowhead) and costoclavicular space (open arrow) (interscalene triangle and costoclavicular compression)

patients with arms in adduction. In abduction, compression of the subclavian artery resulting in varying degrees of stenosis or even complete occlusion has been described. In addition, the most typical form of stenosis is described as band-like or concentric compression $(22,24,26,27)$. Although this method provides the highest resolution in all of imaging modalities currently available, it is an invasive procedure and has a lower but considerable complication rate. Other disadvantages of the method are contraindication in allergic cases, nephrotoxicity of contrast material, and ionizing radiation $(26,29)$. As arterial and venous compression findings present intermittently, the pathology could not be depicted during angiography. Also arteriographic and venographic images, routinely obtained in the antero-posterior projection, are not suitable for demonstration of perpendicular compression that can be seen in TOS $(15,24)$.
As an elevated or relatively elevated position of arms exacerbates symptoms, particularly pain, provocative maneuvers like hyperabduction can be used in some radiologic methods. In this way, alternative diagnostic modalities such as Doppler US, CT angiography, MRI and MRA have been described for a less invasive diagnosis $(3,9,10,15-19,22-24)$. Overlying bony skeleton, obesity and difficulty of differentiation of large collaterals from the subclavian vein were noted as diagnostic difficulties in Doppler US studies. For this reason, duplex scanning of the subclavian vein has been noted to have low sensitivity and specificity (30).

The usefulness of $\mathrm{CT}$ angiography in evaluation of patients with TOS has been suggested, and both complex anatomic relations which include osseous and vascular structures in thoracic outlet as well as the diagnosis of TOS simultaneously have been reported. However, ionizing radiation and the ne- 

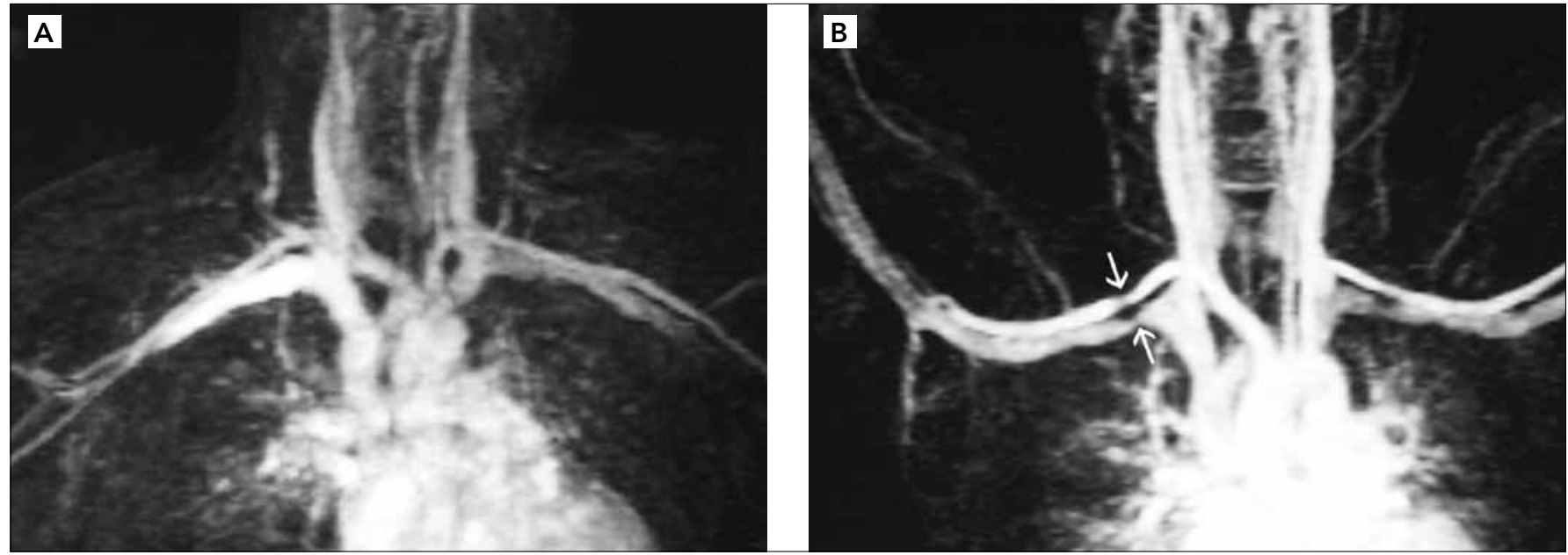

Figure 7. (A) Significant vascular pathology was not seen during arms adduction on the MIP image in equilibrium phase. (B) Compression sign was determined at costoclavicular localization (arrows) during arms abduction particularly in right subclavian vein (costoclavicular compression)
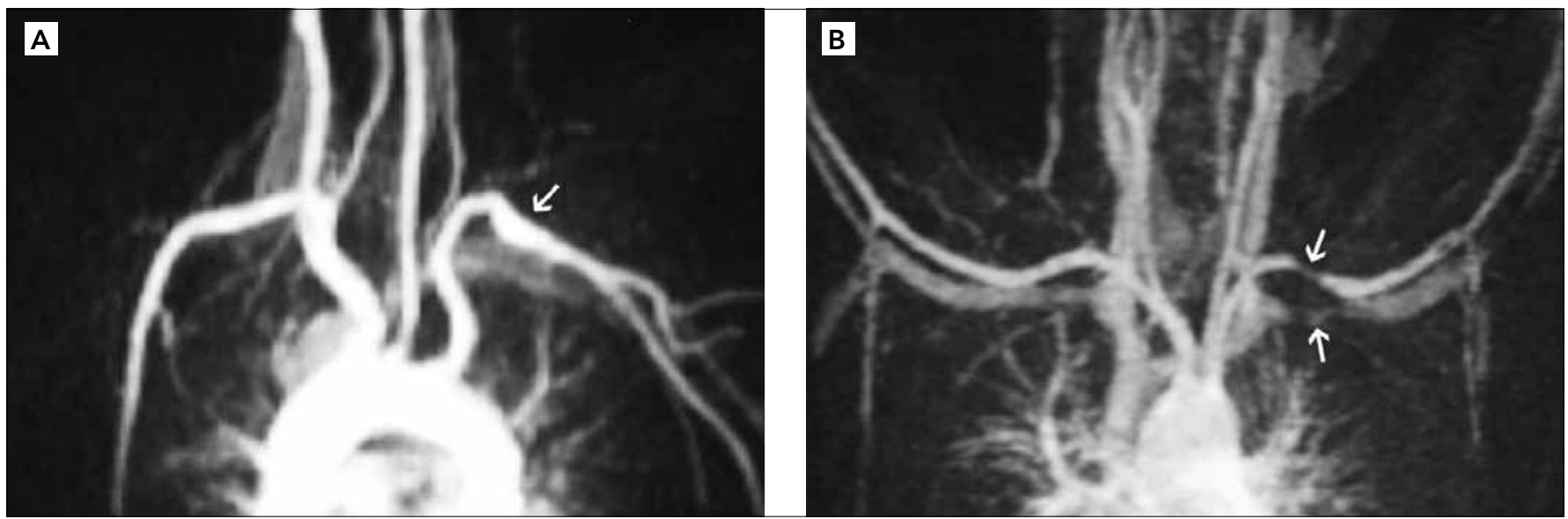

Figure 8. (A) Significant fusiform dilatation is seen in left subclavian artery (solid arrow) during arms adduction by contrastenhanced MRA in arterial phase. (B) Severe compression is detected in left subclavian artery and vein (arrows) during arm abduction by MRA in equilibrium phase (costoclavicular compression)

cessity for iodinated $x$-ray contrast media are potential disadvantages of the technique $(9,17,18)$.

The visibility of both bony anomalies and bandlike structures represents a fundamental advantage of MR imaging over other imaging techniques. Several authors have reported the usefulness of conventional MR sequences on sagittal and coronal planes for demonstrating neurovascular compression and functional anatomy. The difficulty of MRI is that symptoms may occur only in abduction and this position cannot be maintained for a long time during examination $(3,10,19)$. In recent years, there have been some studies on the use of the two-dimensional time-of-flight (TOF) MRA technique in the diagnosis of TOS. However, the main limitations of this technique are the spin dephasing that occurs in the complex or turbulent flow pattern, artifactual signal loss associated with the saturation of slow-flowing blood and long acquisition time that increases the probability of motion and breathing artefact $(12,24)$.

Contrast-enhanced MRA has the potential to overcome some of these problems. It was first described in the early 1990s (31) and has undergone important modifications since the introduction of fast imaging techniques. Contrast enhanced MRA has a higher signal to noise ratio and a shorter acquisition time than other MRA techniques. In addition, due to lack of vascular saturation issues, contrast-enhanced MR angiograms can be acquired in transverse, coronal or sagittal planes. Compared to nonenhanced MRA imaging, such as phase-contrast MR imaging or TOF imaging, contrast-enhanced MRA techniques are less susceptible to artifactual signal loss associated with saturation caused by slow blood flow. The MR signal on contrast enhanced MRA depends on the T1 shortening effect of gadolinium. Motion artefacts are less common because of short acquisition time. Coronal plane enables long segment vessel coverage from the aortic arch to the proximal brachial arteries similar to those obtained by catheter angiography. In comparison with iodinated contrast, on the other hand, gadolinium based contrast agents are not nephrotoxic and provoke anaphylaxis extremely rarely $(25,31,32)$.

The role of CE MRA in evaluation of TOS has only scarcely been mentioned (22-24). According to the preliminary study of Dymarkowski et al. (23), 3D contrast-enhanced MRA has re- 

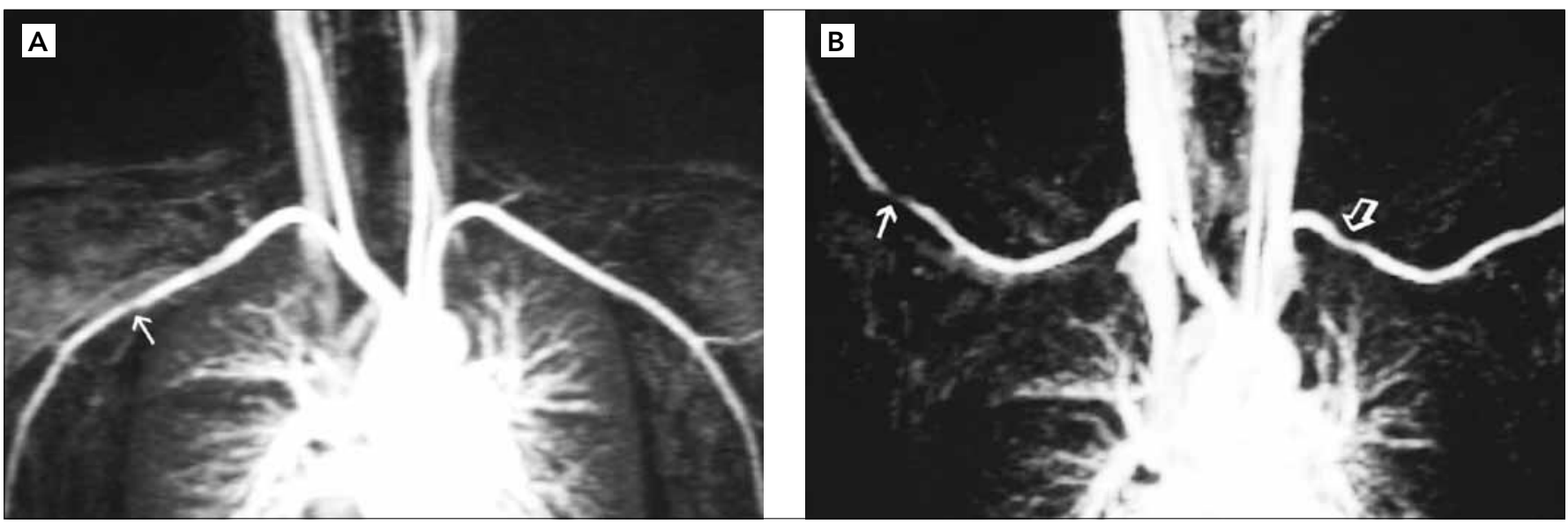

Figure 9. (A) In right subclavian artery at subcoracoid region during adduction, suspicious stenosis sign (solid arrow) is shown in arterial phase MRA image. (B) Stenosis (solid arrow) was more evident during abduction in the same case. Also at left costoclavicular localization in subclavian artery, impingement (open arrow) sign was seen (subcoracoid and costoclavicular compression)

vealed the vessel compression in the interscalene angle and/ or costoclavicular junction during hyperabduction of the arms, whereas the images during adduction showed no vascular pathologies. In this study, the authors concluded that contrastenhanced MRA results are comparable to those of catheter angiography. In another TOS study with MRA, Charon et al. (24) compared 2D TOF sequence with 3D contrast-enhanced MRA. Both MRA sequences may demonstrate significant arterial impingement in this study, but investigators emphasized that 3D contrast-enhanced MRA had some advantages such as extensive vessel coverage and being less prone to artefacts.

Although vascular TOS was reported as $2 \%$ in literature, there was significant arterial compression in more than $60 \%$ in hyperabduction in 2 of 22 cases $(9.09 \%)$ in our study. Also, in 19 of the all 44 arms, impingement or less than $60 \%$ stenosis in hyperabduction was depicted. Six of them had equivocal impingement in adduction. In addition to these findings, focal dilatation was found in 3 subclavian arteries. In 14 of the 44 sides, venous compression coexisted with the arterial lesions, and solated compression was found in 3 arms. In our study, venous overlapping was seen in the first phase of MRA in only 3 cases, but there was no diagnostic difficulty. The high rate of compression findings is probably because our study was planned with selected cases. In our study, subclavian-axillary vessels could be evaluated in different aspects and compression findings were shown by the MIP algorithm composed of contrast enhanced MRA images. As the median age of our patients was 38.8 , incidence of atherosclerotic lesions were quite low in this age group (26). Thus arterial pathologies that were seen were thought to be caused by TOS. Also, location of the vascular signs in special zones like the interscalene triangle, costoclavicular space etc. supports TOS. Limitations of our study is that lesions were not confirmed by catheter angiography, which is valuable in patients with symptoms of microemboli, hand ischemia, Raynaud phenomenon, complaints at rest and in whom surgical intervention is planned, but none of our patients had those symptoms. Also, the diagnostic values of CE-MRA in different arterial systems were comparable with catheter angiography (32).

\section{Conclusion}

The existence of TOS is a cause of much disagreement among clinicians regarding its diagnostic criteria and diverse symptoms and some of the physical examination findings (such as absence or decrease of radial pulses in hyperabduction) could be seen in normal individuals. The diagnosis of TOS must be based on physical examinations and appropriate imaging findings. Other imaging modalities besides CEMRA have various limitations such as invasiveness, ionizing radiation, nephrotoxicity, iodinated contrast media reactions, obesity or skletal limitations. CE-MRA is a rapid (total imaging time is approximately $20 \mathrm{~min}$ ), reliable, repeatable (allowing assesment of the vasculature in adduction and abduction during one examination) and noninvasive vascular imaging method which is independent of the above limitations. Another advantage of this method is screening of both arterial and venous systems during different phases. According to our study, the imaging diagnosis of the vascular and neurovascular TOS can be made with 3D contrast-enhanced MRA as a scanning or early diagnostic method. Thus, by applying invasive methods such as catheter angiography in only selected patients, we can prevent unnecessary invasive interventions and possible complications.

\section{Conflict of Interest}

No conflict of interest was declared by the authors.

\section{References}

1. Roos DB, Owens JC. Thoracic outlet syndrome. Arch Surg 1966;93:71-4. [CrossRef]

2. Novak CB, Mackinnon SE. Thoracic outlet syndrome. Orthop Clin North Am 1996;27:747-62.

3. Demondion X, Bacqueville E, Paul C, Duquesnoy B, Hachulla E, Cotten A. Thoracic outlet: assessment with MR imaging in asymptomatic and symptomatic populations. Radiology 2003;227:4618. [CrossRef]

4. Wilbourn AJ. The thoracic outlet syndrome is overdiagnosed. Arch Neurol 1990;47:328-30. 
5. Davidovic LB, Kostic DM, Jakovljevic NS, Kuzmanovic IL, Simic TM Vascular thoracic outlet syndrome. World J Surg 2003;27:545-50. [CrossRef]

6. Roos DB. The thoracic outlet syndrome is underrated. Arch Neurol 1990;47:327-30.

7. Huang JH, Zager EL. Thoracic outlet syndrome. Neurosurgery 2004;55:897-903. [CrossRef]

8. Mackinnon SE, Novak CB. Thoracic outlet syndrome. Curr Probl Surg 2002;39:1070-145. [CrossRef]

9. Matsumura JS, Rilling WS, Pearce WH, Nemcek AA Jr, Vogelzang $\mathrm{RL}$, Yao JS. Helical computed tomography of the normal thoracic outlet. J Vasc Surg 1997;26:776-83. [CrossRef]

10. Panegyres PK, Moore N, Gibson R, Rushworth G, Donaghy M. Thoracic outlet syndromes and magnetic resonance imaging. Brain 1993;116:823-41. [CrossRef]

11. Tateishi A. Diagnosis and management of thoracic outlet syndrome. Nippon Seikeigeka Gakkai Zasshi 1980;54:817-27.

12. Ohkawa $Y$, Isoda $H$, Hasegawa $S$, Furuya $Y$, Takahashi $M$, Kaneko M. MR angiography of thoracic outlet syndrome. J Comput Assist Tomogr 1992;16:475-7. [CrossRef]

13. Seror P. Frequency of neurogenic thoracic outlet syndrome in patients with definite carpal tunnel syndrome: an electrophysiological evaluation in 100 women. Clin Neurophysiol 2005;116:259-63. [CrossRef]

14. Ide J, Kataoka Y, Yamaga M, Kitamura T, Takagi K. Compression and stretching of the brachial plexus in thoracic outlet syndrome: correlation between neuroradiographic findings and symptoms and signs produced by provocation manoeuvres. J Hand Surg $\mathrm{Br}$ 2003;28:218-23. [CrossRef]

15. Longley DG, Yedlicka JW, Molina EJ, Schwabacher S, Hunter DW, Letourneau JG. Thoracic outlet syndrome: evaluation of the subclavian vessels by color duplex sonography. AJR Am J Roentgenol 1992;158:623-30. [CrossRef]

16. Wadhwani R, Chaubal N, Sukthankar R, Shroff M, Agarwala S. Color Doppler and duplex sonography in 5 patients with thoracic outlet syndrome. J Ultrasound Med 2001;20:795-801. [CrossRef]

17. Remy-Jardin M, Doyen J, Remy J, Artaud D, Fribourg M, Duhamel A. Functional anatomy of the thoracic outlet: evaluation with spiral CT. Radiology 1997;205:843-51. [CrossRef]

18. Remy-Jardin M, Remy J, Masson P, Bonnel F, Debatselier P, Vinckier L. CT angiography of thoracic outlet syndrome: evaluation of imaging protocols for the detection of arterial stenosis. J Comput Assist Tomogr 2000;24:349-61. [CrossRef]

19. Demondion X, Boutry N, Drizenko A, Paul C, Francke JP, Cotten A. Thoracic outlet: anatomic correlation with MR imaging. AJR Am J Roentgenol 2000;175:417-22. [CrossRef]
20. van Es HW. MRI of the brachial plexus. Eur Radiol 2001;11:325-36. [CrossRef]

21. Yanaka K, Asakawa H, Matsumaru Y, Kujiraoka Y, Nose T. Diagnosis of vascular compression at the thoracic outlet using magnetic resonance angiography. Eur Neurol 2004;51:122-3. [CrossRef]

22. Hagspiel KD, Spinosa DJ, Angle JF, Matsumoto AH. Diagnosis of vascular compression at the thoracic outlet using gadolinium-enhanced high-resolution ultrafast MR angiography in abduction and adduction. Cardiovasc Intervent Radiol 2000;23:152-4. [CrossRef]

23. Dymarkowski S, Bosmans H, Marchal G, Bogaert J. Three-dimensional MR angiography in the evaluation of thoracic outlet syndrome. AJR Am J Roentgenol 1999;173:1005-8.

24. Charon JP, Milne W, Sheppard DG, Houston JG. Evaluation of MR angiographic technique in the assessment of thoracic outlet syndrome. Clin Radiol 2004;59:588-95. [CrossRef]

25. Cosottini M, Zampa V, Petruzzi P, Ortori S, Cioni R, Bartolozzi C. Contrast-enhanced three-dimensional MR angiography in the assessment of subclavian artery diseases. Eur Radiol 2000;10:1737-44. [CrossRef]

26. Lang EK. Arteriography of thoracic outlet syndrome. In: Baum S, editor. Abrams angiography: vascular and interventional radiology. Boston: Little, Brown Medical Division; 1997. p. 995-1010.

27. Kadir S. Arteriography of the upper extremities. In: Kadir S, editor. Diagnostic angiography. Philadelphia: WB Saunders; 1986. p. 172-206.

28. Degeorges R, Reynaud C, Becquemin JP. Thoracic outlet syndrome surgery: long-term functional results. Ann Vasc Surg 2004;18:558-65. [CrossRef]

29. Connors JJ 3rd, Sacks D, Furlan AJ, Selman WR, Russell EJ, Stieg $\mathrm{PE}$, et al. Training, competency, and credentialing standards for diagnostic cervicocerebral angiography, carotid stenting, and cerebrovascular intervention: a joint statement from the American Academy of Neurology, American Association of Neurological Surgeons, American Society of Interventional and Therapeutic Radiology, American Society of Neuroradiology, Congress of Neurological Surgeons, AANS/CNS Cerebrovascular Section, and Society of Interventional Radiology. Radiology 2005;234:26-34. [CrossRef]

30. Haire WD, Lynch TG, Lund GB, Lieberman RP, Edney JA. Limitations of magnetic resonance imaging and ultrasound-directed (duplex) scanning in the diagnosis of subclavian vein thrombosis. J Vasc Surg 1991;13:391-7. [CrossRef]

31. Prince MR. Gadolinium-enhanced MR aortography. Radiology 1994;191:155-64.

32. Yang CW, Carr JC, Futterer SF, Morasch MD, Yang BP, Shors SM, et al. Contrast-enhanced MR angiography of the carotid and vertebrobasilar circulations. AJNR Am J Neuroradiol 2005;26:2095-101. 